\section{$\underset{\text { hommes }}{\text { \& migrations }}$}

\section{Hommes \& migrations}

Revue française de référence sur les dynamiques

migratoires

1308 | 2014

Les Paris des migrants

\title{
La Maison des journalistes de Paris
}

Abriter les héros en fuite

\section{Sophie Pasquet}

\section{OpenEdition \\ 1 Journals}

\section{Édition électronique}

URL : http://journals.openedition.org/hommesmigrations/3030

DOI : 10.4000/hommesmigrations.3030

ISSN : 2262-3353

\section{Éditeur}

Musée national de l'histoire de l'immigration

\section{Édition imprimée}

Date de publication : 1 octobre 2014

Pagination : 170-173

ISBN : 978-2-919040-29-2

ISSN : $1142-852 X$

\section{Référence électronique}

Sophie Pasquet, "La Maison des journalistes de Paris », Hommes \& migrations [En ligne], 1308| 2014 mis en ligne le 26 juin 2015, consulté le 24 septembre 2020. URL : http://journals.openedition.org/ hommesmigrations/3030; DOI : https://doi.org/10.4000/hommesmigrations.3030 


\section{LA MAISON}

DES JOURNALISTES

DE PARIS

\section{ABRITER LES HÉROS EN FUITE}

SOPHIE PASQUET, photojournaliste, membre du collectif HANS LUCAS

A u 15 de la rue de Cauchy, à Paris, on ressent immédiatement un mélange de calme et de gravité, de clarté et de protection. Sans doute estce dû aux murs épais et aux larges fenêtres en verre dépoli qui laissent passer la lumière sans rien laisser voir de l'intimité. Dans la large entrée accueillante, une plaque en mémoire d'Anna Politkovskaïa, journaliste russe assassinée à Moscou le 7 octobre 2006.

Depuis 2002, la Maison des journalistes (MDJ) accueille pour six mois des journalistes persécutés dans leur pays. 14 chambres sont à leur disposition. Deux sont équipées de sanitaires intérieurs pour ceux souffrant de handicaps physiques ou victimes de tortures. Quand elles sont libres, on les réserve aux femmes qui représentent un tiers des journalistes hébergés. II y passe peu de couples, jamais d'enfants. Ici, c'est l'urgence que l'on gère.

Le début de l'histoire de la Maison des journalistes est la rencontre d'une belle âme, Danièle Ohayon, avec un destin épique. L'ancienne journaliste sourit en se souvenant du déclic : "Pour France Info, j'interviewais souvent des journalistes réfugiés. Chez eux, ils étaient des héros: ils avaient dix ans de métier et ils étaient souvent connus pour avoir été persécutés. Mais en arrivant à Paris, ils n'étaient plus rien. Ils se retrouvaient à la rue ou à dormir sur le canapé d'une connaissance. Ici, les héros devenaient transparents." Si on lui demande un nom, sans hésiter, c'est celui de Faraj Sarkouhi, journaliste iranien qu'elle interviewe en 1999 à la descente de l'avion : Issu d'une famille très modeste, il a commencé en balayant les locaux de l'imprimerie. II a grimpé tous les échelons petit à petit et il a fini par être envoyé à l'université. Suite à des manifestations, il a été emprisonné dix ans sous le Chah. Il a trente ans quand Khomeiny arrive au pouvoir en 1979. II lance une revue culturelle. II retourne en prison car il publie la photo d'un jeune couple se tenant par la main. Sa famille réussit à s'exiler mais il est arrêté à l'aéroport, remis en prison, puis relâché. Une nuit, il fracasse la vitrine d'une agence de voyages pour pouvoir envoyer un fax à sa femme, réfugiée en Allemagne. II a 60 ans quand il arrive finalement à Paris grâce à Reporters sans frontières. À la fin de l'interview, je lui demande: 'Vous allez dormir où ce soir ?' Je vois que sa femme pleure à ses côtés. Je comprends alors 


\section{Un lieu d'accueil pour les journalistes persécutés}

Aujourd'hui, c'est Darline Cothière, devenue directrice en 2011, qui fait visiter la maison de trois étages. Elle a un rire clair qui résonne dans les couloirs, malgré les difficultés. Sur la porte de chaque chambre est écrit le nom du média qui la parraine. Signe de la baisse des subventions, il y a parfois deux noms sur une même porte... Aujourd'hui, la MDJ fonctionne avec une équipe de quatre personnes : sa directrice, un assistant social, une chargée de mission qui s'occupe de L'Fil de l'exilé, le journal en ligne, et un gardien de nuit. Économies obligent, les cours de français quotidiens et les séances de la psychologue deux fois par

Darline Cothière, directrice de la Maison des Journalistes (c) Sophie Pasquet - hanslucas.com

qu'ils n'en savent rien. Je décide de lancer cette idée qui me trotte dans la tête depuis longtemps: un lieu d'accueil."

Danièle Ohayon monte le projet avec Philippe Spineau, bon copain, ancien éducateur, réalisateur, "quelqu'un d'incroyablement humain sans être complaisant". La Mairie de Paris leur cède une ancienne usine à brosses en 2004. Un lieu de $800 \mathrm{~m}^{2}$ à rénover. Pour faire vivre leur idée, ils sollicitent l'aide des médias français. "C'était inhérent à notre projet : pour que ces journalistes en fuite retrouvent leur dignité, ils avaient besoin de la reconnaissance de la profession. On a demandé aux médias de parrainer chacun une chambre pour 10 ooo euros par an." Le Monde dit "oui" en premier. Sud-Ouest, Ouest-France, Radio-France, RFI, Le Canard enchaîné, TF1, Les Échos, Arte, M6, Canal-Plus, Capa, Paris Match, Bayard Presse le rejoindront. La MDJ devient alors une structure unique... au monde. Elle l'est toujours. mois reposent sur des bénévoles.

"Pour personne, fuir n'est un projet de vie. Rien n'a été réfléchi. La cassure est très brutale. Ces personnes ont d'abord besoin d'une chambre où elles se sentent en sécurité mais aussi d'activités et d'espaces communs où se retrouver et échanger sur l'exil", explique-t-elle en présentant la cuisine et les frigos partagés, la large salle à manger décorée de dessins de presse, la bibliothèque qui sert aussi de salle de télévision. Avant, le wifi n'était installé que dans les parties communes, pour pousser les résidents à se retrouver malgré la tentation de l'isolement, l'obstacle de la langue et les désaccords politiques parfois... Maintenant, il fonctionne dans toutes les chambres. Le profil de ces journalistes aussi a changé. "Nous accueillons de plus en plus d'activistes', c'est-à-dire des jeunes qui n'ont pas forcément de carte de presse mais qui ont fait un énorme travail d'information, comme les bloggueurs des révolutions arabes ou ceux qui ont filmé des exactions. Ils sont souvent à la fois journalistes, ingénieurs ou étudiants, sociologues ou écrivains." Ils sont adressés par France Terre d'Asile, Reporters sans frontières, les 
Maria A. a 24 ans. Elle est journaliste syrienne. A la Maison des Journalistes, elle prend des cours de Français et rêve de retrouver son métier. Elle attend son statut de réfugiée politique $\odot$ SophIE PASQUet - hansLuCAS.com

ambassades de France de leur pays d'origine, des confrères français avec lesquels ils ont travaillé en tant que "fixeurs"... Aujourd'hui, ils sont syriens, afghans, guinéens, tibétains, rwandais, ivoiriens... "Les preuves de leur activité de journalistes sont souvent tangibles. On les trouve sur internet, sur YouTube ou leur blog, ou bien ils arrivent avec leurs propres articles de presse signés", explique la directrice. Elle passe aussi des coups de fil pour vérifier un parcours, un témoignage, et pour faire sa propre enquête. Darline l'évoque rarement mais cette ancienne professeure de français langue étrangère est aussi une ancienne réfugiée politique, partie d'Haïti en 2001.

À 24 ans, Maria a déjà deux vies : celle d'avant la guerre et celle d'après. Elle est toute jeune jour- naliste quand elle est obligée de quitter son pays, la Syrie en guerre, pour avoir pris la défense de confrères emprisonnés. Elle prend la direction du Liban, puis d'Istanbul, où elle retrouve sa famille partie par d'autres chemins, puis c'est l'arrivée à Aman en Jordanie : "J'y suis restée un an et demi. J'ai pu travailler pour une chaîne irakienne et puis les menaces de l'État islamique ont repris. Quand j'ai compris qu'ils mavaient retrouvée, je ne pouvais plus travailler. Ma mère est partie en Algérie avec mon frère. Je suis arrivée en France avec mon père." Chez elle, tout le monde baignait dans la culture française. C'est à l'ambassade à Aman qu'elle demande l'asile. Voilà trois mois qu'elle est à Paris, deux semaines à la Maison des journalistes. "CC'a été difficile d'entrer ici, la liste d'attente est longue. Je suis très heureuse. On est six journalistes syriens mais j'essaie de ne pas parler seulement avec eux. Je veux apprendre le français et retravailler le plus vite possible." Elle a de longs cheveux blonds, de l'énergie à revendre... et des yeux qui s'embuent malgré son sourire. Elle attend son rendez-vous à l'Office français de protection des réfugiés et apatrides (Ofpra) pour avoir son statut de réfugiée, premier sésame de sa deuxième vie, "avec inquiétude mais dans le calme, je n'ai plus peur... si ce n'est de l'avenir".

Depuis sa création, plus de $95 \%$ des journalistes accueillis à la MDJ obtiennent le statut de réfugié (en 2013, en France, 24\% des demandes ont été satisfaites). Souvent, ils ont des preuves de leurs persécutions, on en a parlé chez eux, dans les journaux ou sur internet ... et ils sont préparés à l'entretien à l'Ofpra. Le plus gros écueil est quand ils viennent d'un pays inscrit sur la liste des "pays sûrs" de l'Ofpra, comme Roman, photographe bangladais qui n'a jamais obtenu son statut, alors que le Bangladesh a, depuis, été retiré de la liste... “Aujourd'hui, on prépare davantage ce qui va se 
passer après l'obtention du statut et quand ils vont devoir quitter la MDJ, explique Darline Cothière. Dans un premier temps, on les aide à se reconstruire, à comprendre l'administration française, à obtenir le statut, puis au bout de quatre mois, ils signent un contrat d'insertion où l'on travaille sur le projet de travail et d'hébergement."

\section{Préparer la vie d'après}

Moins de $10 \%$ de ces journalistes étrangers retrouveront leur métier. En question : l'écueil de la langue, la crise dans la presse française, mais aussi une grande frilosité des médias envers des journalistes qui ne ressemblent pas à leurs confrères français. La MDJ multiplie les partenariats pour valoriser leurs compétences : grâce au journal en ligne L'EFil de l'exilé, ils continuent à écrire, photographier, enquêter, interviewer... Ils réalisent des expositions, témoignent auprès des lycéens, participent au festival de Bayeux des correspondants de guerre, font profiter les médias parrains de leur expertise sur leur pays... Mais ensuite, c'est l'hôtellerie, la restauration ou les postes de vigile qui leur permettent de gagner leur vie.

Chérif est guinéen. Il voudrait mettre à profit son passé de jeune sociologue pour travailler dans l'humanitaire et retourner un jour en Afrique. Après avoir longtemps "fait le 115", il est à la Maison des journalistes depuis cinq mois : le départ approche. Il attend encore son statut de réfugié et ne sait pas où il sera hébergé ensuite. À Conakry, il avait monté son propre journal, Jeune presse, qu'il a dû fermer quand l'opérateur de téléphonie qui achetait sa dernière page a retiré ses billes. Lors des élections législatives de 2013, il dénonce des irrégularités et il se retrouve en prison. "Je savais que j'allais y rester... En Guinée, on 'oublie' les gens en prison. J'ai pu m'échapper grâce au personnel hospitalier. Je me suis dit que je n'avais pas le choix." II le répète souvent, comme si la question torturait encore son esprit. Derrière le jeune homme de 34 ans, on devine le petit garçon déjà épris de vérité et de justice que tentait de calmer son père fonctionnaire, âgé de 86 ans aujourd'hui. Chérif ne sait pas s'il le reverra. II touche 345 euros d'allocations au titre de demandeur d'asile. La MDJ lui fournit un titre de transport, des tickets restaurant, l'a aidé à obtenir la couverture maladie universelle (CMU), l'inscrip-
Shérif S.D. a 34 ans. Il est journaliste Guinéen. Mis en prison pour avoir dénoncé des irrégularités lors des élections de 2013, il a du prendre la fuite (c) SOPHIE PASQUet - hansLuCAS.COM

tion à Pôle emploi... II dit qu'il a tout perdu, y compris sa bourse d'études au Canada. La nuit, il travaille sans relâche sur son ordinateur, intervient sur des blogs, écrit ses propres articles. "Quelquefois, je reçois encore des menaces! Mais ici, je suis plus fort pour continuer le chemin et transformer mon pays. Là bas, les gens ne comprennent pas que, si je le critique, c'est parce que je l'aime." 